\title{
Pratiques
}

Linguistique, littérature, didactique

\section{Les péripéties du prédicat en Suisse romande : hier, aujourd'hui et demain...}

Martine Panchout-Dubois, Roxane Gagnon et Ecaterina Bulea Bronckart

\section{(2) OpenEdition}

\section{Journals}

Édition électronique

URL : http://journals.openedition.org/pratiques/3765

DOI : 10.4000/pratiques.3765

ISSN : 2425-2042

Éditeur

Centre de recherche sur les médiations (CREM)

Référence électronique

Martine Panchout-Dubois, Roxane Gagnon et Ecaterina Bulea Bronckart, «Les péripéties du prédicat en Suisse romande : hier, aujourd'hui et demain... », Pratiques [En ligne], 175-176 | 2017, mis en ligne le 22 décembre 2017, consulté le 29 avril 2019. URL : http://journals.openedition.org/pratiques/3765 ; DOI : 10.4000/pratiques.3765

Ce document a été généré automatiquement le 29 avril 2019.

(c) Tous droits réservés 


\section{Les péripéties du prédicat en Suisse romande : hier, aujourd'hui et demain...}

Martine Panchout-Dubois, Roxane Gagnon et Ecaterina Bulea Bronckart

Mais celui qui écrit pour l'enfance doit avoir l'éducation de l'esprit et du cœur en vue, et régler sur ce but la marche et la forme de l'enseignement.

Il faut que la méthode soit rigoureusement progressive, et que les élèves, du commencement à

la fin, s'aident à composer eux-mêmes leur grammaire. Ainsi, loin de faire des recueils sur le nom, l'adjectif, le verbe, etc., et de réunir sur ces

parties ce qui les concerne, il faut s'attacher au fond du langage, commencer par le

commencement, aller pas à pas du simple au composé, et apprendre aux enfants à penser, pour leur apprendre à comprendre et à parler la langue de l'homme. Les menus détails ne peuvent paraître

que plus tard et par occasion. De là résulte nécessairement un déplacement des matières grammaticales que l'on s'est occupé à réunir et à rapprocher. De là encore une grande parcimonie dans les définitions et les divisions abstraites qui rebutent l'enfance, parce qu'elle ne peut pas encore s'élever aussi haut. Il y en a d'ailleurs qui ne présentent aucune utilité. Une autre chose importante dans un cours éducatif de langue, c'est d'associer constamment les élèves

à la création du langage [...] Grégoire Girard (1876, p. 14) 
1 L'usage du terme de prédicat dans la réalité des classes romandes ne va pas de soi.

2 Son relatif non-usage illustre un problème récurrent de la grammaire scolaire: le flottement terminologique (Vargas, 1995 ; Chartrand \& de Pietro, 2010) ; d'autres notions de la grammaire scolaire sèment leur petit lot de controverses: les notions de classe, celles de groupe, de mode, de personne, de complément, de phrase, de grammaire... L'une des tâches centrales de la didactique consiste à étudier les transformations des objets d'enseignement (Schneuwly \& Dolz, 2009), il est donc particulièrement intéressant de se demander pourquoi le terme de prédicat est peu usité dans les pratiques d'enseignement.

3 Ce texte, qui illustre notre point de vue de formatrices engagées dans la formation initiale et continue d'enseignant.e.s du primaire et du secondaire 1 en Suisse romande, propose d'abord une brève analyse des différentes acceptions du terme de prédicat. Il présente ensuite des éléments de l'histoire de la rénovation de l'enseignement grammatical en Suisse romande, suivi d'un état des lieux ayant trait au contexte et aux pratiques d'enseignement contemporaines relatifs à l'usage du terme de prédicat. Pour compléter cet état des lieux, nous avons conduit une enquête auprès des étudiant.e.s du Bachelor en enseignement primaire à la Haute école pédagogique Vaud (HEP Vaud) ainsi qu'auprès de responsables de formation ou de personnes impliquées dans la conception des documents institutionnels (plan d'études, mémentos grammaticaux ou moyens d'enseignement). Notre texte met ensuite en évidence les raisons qui fondent l'adoption du terme dans le cadre d'une grammaire scolaire, puis il énonce les raisons de la nécessité de son usage dans le cadre de l'enseignement grammatical, tout en précisant ses limites. Enfin, ce texte esquisse quelques propositions qui viseraient à en faciliter l'usage systématique dans les classes.

\section{Des diverses acceptions du terme de prédicat}

Pour comprendre les diversités et nuances des sens donnés au terme de prédicat et reprendre conscience des usages du terme dans le temps, en fonction des approches grammaticales différentes, parallèles ou successives, il nous faut d'abord regarder ces reformulations successives au cours de l'histoire. Le terme n'est pas nouveau. Ainsi que l'expose clairement P. Le Goffic (1993, p. 13), dans la tradition classique, à la suite de Platon et d'Aristote, «les deux termes de la phrase se définissent mutuellement et circulairement. Le sujet est ce dont on prédique, le prédicat est ce qu'on prédique; le prédicat lui-même s'analyse en copule (être) + attribut ». Cette analyse, d'essence logique, a perduré pendant des siècles et il faut attendre le $\mathrm{XX}^{\mathrm{e}}$ siècle pour que naisse la grammaire des fonctions. C'est de l'analyse éclatée du prédicat que proviennent l'attribut - au sens qui est le sien aujourd'hui -, le complément d'objet direct, indirect et second, le complément circonstanciel.

5 Il est intéressant de souligner au passage, que «cette grammaire des fonctions, plus récente qu'on ne l'imagine souvent, ne couvre qu'une partie des faits de relation dans la phrase : des pans entiers du fonctionnement de la langue ne sont pas couverts par une quelconque dénomination de fonction » (ibid.) ${ }^{1}$. Toutefois, l'articulation de la structure logico-grammaticale avec l'analyse en fonctions peut se représenter ainsi : 
Tableau . Reproduction de la schématisation de la structure logico-grammaticale proposée par P. Le Goffic (1993, p. 13)

\begin{tabular}{|l|l|l|l|l|}
\hline sujet & \multicolumn{2}{|l|}{ prédicat } & éléments extra-prédic. \\
\hline sujet & verbe & compl. essentiels & compl. access. intra-prédic. & $\begin{array}{l}\text { compl. access. } \\
\text { extra-prédic. }\end{array}$ \\
\hline
\end{tabular}

Ce tableau montre que la notion de prédicat conserve sa validité et son intérêt logique et reste au cœur de l'analyse de la phrase à condition de reconnaitre l'existence d'éléments intra- ou extra-prédicatifs, dont la frontière est souvent difficile à situer. Aujourd'hui, la phrase se définit communément comme une séquence autonome qui met en relation deux termes obligatoires, un sujet et un prédicat, termes auxquels s'adjoignent d'éventuels constituants facultatifs.

7 Historiquement, cette structuration logico-grammaticale a tardé à être distinguée de l'opposition thème/rhème, d'ordre plus psychologique (Le Goffic, 1993). En effet, la distinction n'est effective qu'à la fin du XIX siècle : le thème désigne ce dont l'énoncé parle et qui est déjà connu tandis que le rhème désigne ce qui est dit du thème et constitue donc une information nouvelle. Il est possible de remarquer que dans les phrases canoniques, il y a souvent superposition de ces deux niveaux de structuration de la phrase. Aujourd'hui, la relation sujet/prédicat est utilisée dans le cadre de l'analyse syntaxique, tandis que l'opposition thème/rhème relève de l'analyse propre à la grammaire textuelle.

Pour préciser encore l'usage ou le non-usage du terme de prédicat dans les divers courants de l'analyse grammaticale, il faut remarquer, à la suite de J.-Ch. Pellat (2011, p. 89), qu'il existe différents modèles d'analyse de la phrase verbale : « la phrase verbale est l'objet de deux représentations concurrentes ». L'une place le verbe au centre de la phrase, l'autre analyse la phrase en deux constituants. Lorsque le verbe est analysé comme le centre de la phrase, comme son noyau, le verbe est la clé de voute de la phrase, dont dépendent le sujet et les compléments. Point n'est alors besoin du terme de prédicat. Lorsque la phrase est analysée en deux constituants essentiels, la phrase verbale canonique comporte le groupe sujet et le groupe verbal. Il peut alors être nécessaire de "désimbriquer » classes et fonctions et d'introduire le terme de prédicat. Cette dernière remarque s'applique très précisément à la situation de la Suisse romande. Pendant des années, les appellations groupe nominal sujet - GNS - et groupe verbal ont provoqué chez les élèves et les enseignant.e.s une confusion entre la catégorie et la fonction.

\section{État des lieux de l'enseignement grammatical en Suisse romande ; point, en particulier, sur les usages qui sont faits du terme de prédicat}

Pour comprendre l'usage du terme de prédicat dans les prescriptions et dans les pratiques, nous esquissons quelques repères historiques de l'enseignement grammatical en Suisse romande; nous examinons le traitement du terme dans les prescriptions officielles et 
rendons compte des principaux résultats d'une enquête auprès d'étudiant.e.s, de responsables de formation et de concepteurs de programme.

\section{Brève mise en perspective historique}

10 La Suisse romande a entrepris, dans le courant des années 1970, une profonde rénovation de l'enseignement grammatical qui s'inscrivait dans le droit fil des propositions didactiques formulées dans le Plan Rouchette publié en France en 1971. Ce plan visait en particulier à mettre en œuvre le principe d'articulation d'un pôle de «libération » fait d'activités visant à l'enrichissement des capacités d'expression orale et écrite, et d'un pôle de "structuration» fait d'activités de réflexion guidée visant à la construction de connaissances relatives aux régularités d'organisation de la langue. Au plan notionnel, la "grammaire rénovée » romande s'est inspirée des principes d'analyse de la linguistique structurale de L. Bloomfield et de la Grammaire générative et transformationnelle de N. Chomsky, et a proposé, dans l'ouvrage princeps Maîtrise du français (Besson et al., 1979), un corpus de notions qui rompaient avec les approches traditionnelles engluées dans des considérations référentielles et sémantiques inextricables (cf. les 29 nuances de compléments circonstanciels répertoriées dans Le Bon usage [Grévisse, 1980]). Ce corpus notionnel de référence visait aussi à clairement différencier les niveaux syntaxique, morphologique et sémantique de l'analyse des unités de la langue, et à différencier tout aussi nettement les niveaux des classes grammaticales (nom, verbe, adjectif, préposition, etc.), des groupes (groupe nominal, groupe verbal, groupe adjectival, groupe prépositionnel) et des fonctions syntaxiques (sujet, prédicat, complément de phrase, complément du nom, etc.).

11 Dans la perspective ci-dessus évoquée, la notion de prédicat a tout naturellement été introduite dans le corpus des notions grammaticales, au titre de fonction syntaxique assurée par le groupe verbal:

En structure profonde, le groupe nominal qui précède le groupe verbal est constituant obligatoire de la phrase. On dit qu'il est sujet de la phrase. Le groupe verbal est aussi constituant obligatoire de la phrase. On dit qu'il est prédicat de la phrase. Le groupe verbal comprend un verbe qui entretient un rapport formel avec le groupe nominal sujet. (Besson et al., 1979, p. 341)

Dans le canton de Genève, cette notion a d'emblée été introduite dans le corpus notionnel proposé aux enseignant.e.s primaires en formation, au titre de l'une des trois fonctions syntaxiques majeures (cf. Bronckart \& Sznicer, 1990), et elle a conservé jusqu’à ce jour ce statut dans les documents destinés à la formation, comme en atteste l'ouvrage récent de Bulea Bronckart (2015).

En dépit des principes initiaux de la rénovation romande, et en dépit de la teneur des corpus notionnels proposés dans certains dispositifs de formation, la notion de prédicat ne s'est néanmoins véritablement imposée ni dans les autres moyens d'enseignement en usage en Suisse romande, ni dans les pratiques d'enseignement et de formation, comme le montrent les études relatées ci-dessous.

\section{Traitement du terme prédicat dans les prescriptions romandes}

La perspective romande assigne à l'enseignement grammatical à l'école une double visée : d'une part, doter les élèves de savoirs relatifs au fonctionnement de la langue dans le but 
de les aider à lire et, surtout, à écrire ; d'autre part, permettre aux élèves de construire une représentation de la langue en tant que système. C'est dans cette dernière visée, qui implique cohérence et systématicité, que s'ancrent les arguments en faveur du prédicat. Mais qu'en est-il du prédicat dans les diverses instructions officielles, à savoir le plan d'études romand (désormais PER), la grammaire de référence mise à disposition des enseignant.e.s Lire, écrire, comprendre la grammaire et la langue (Balma, Tardin \& de Pietro, 2013), les mémentos Texte et Langue (Aubert \& Messeiller, 2015 ; Balma \& Roduit, 2010), pensés pour les $2^{\mathrm{e}}$ et $3^{\mathrm{e}}$ cycles $^{2}$, les moyens d'enseignement romands (désormais MER)?

Le PER, publié en 2010, est organisé de manière identique pour les trois cycles consécutifs de l'école obligatoire. Il est pensé de manière longitudinale, en adoptant pour chaque champ disciplinaire une organisation en axes thématiques identiques pour chacun des trois cycles. Dans le domaine de l'enseignement du français, inclus dans celui des Langues, le PER propose huit axes thématiques auxquels appartient l'axe du fonctionnement de la langue. Différentes rubriques assurent une logique organisée des apprentissages à mener dans les classes: Grammaire de la phrase, Orthographe, Vocabulaire, Conjugaison et De la Phrase au Texte.

S'agissant de la Grammaire de la phrase, des sous-rubriques relatives aux constituants, aux classes grammaticales et aux fonctions sont repérables pour les trois cycles. Il est fort intéressant de constater que le terme de prédicat n'apparait qu'au $3^{\mathrm{e}}$ cycle sous l'intitulé Analyse de la phrase en constituants obligatoires (sujet, prédicat) et facultatifs (complément de phrase) (CIIP, 2010). Au $2^{\mathrm{e}}$ cycle, il est fait mention de la nécessité de "repérer et d'identifier les constituants obligatoires et facultatifs de la phrase» (p.X) sans que le terme de prédicat soit cité. Il est question, dans la rubrique Les fonctions, identification des fonctions grammaticales, d'identifier le Groupe verbal (GV) sous forme de :

Tableau . Extrait de PER, L1 26

\begin{tabular}{|ll|}
\hline Les fonctions - Identification des fonctions grammaticales & \\
Identification du Sujet sous forme de: & Identification du Sujet sous forme de: \\
Groupe nominal - GN & Groupe nominal - GN \\
Pronom - Pro & Pronom - Pro \\
Nom - N & Nom - N \\
& Infinitif - Inf \\
\hline Identification du Groupe verbal - GV - sous forme de: & Identification du Groupe verbal - GV - sous forme de: \\
Verbe - V & Verbe - V \\
Verbe + Groupe nominal (avec ou sans préposition) - & Verbe + Groupe nominal (avec ou sans préposition) - \\
V + GN (avec ou sans préposition) & V + GN (avec ou sans préposition) \\
& Verbe + Groupe nominal (avec ou sans préposition) \\
& + Groupe nominal (avec ou sans préposition) - V + GN \\
& avec ou sans préposition + GN (avec ou sans preposition) \\
\hline
\end{tabular}

$17 \mathrm{Au} 1^{\mathrm{er}}$ cycle, les mêmes choix terminologiques sont observables : il s'agit, dans la seconde partie du cycle, de repérer les constituants obligatoires et les constituants facultatifs.

18 Pour comprendre ces prescriptions, il faut rappeler que des commissions spécifiques à chaque cycle, constituées chacune de praticiens et d'experts, ont fonctionné de manière à la fois autonome et interdépendante. Il est possible de supposer que les priorités des personnes engagées dans les différentes commissions ont prévalu sans toutefois exclure l'hypothèse d'une décision concertée. Le témoignage d'un expert ayant participé à l'élaboration du programme du $3^{\mathrm{e}}$ cycle va d'ailleurs dans ce sens : il semble qu'un certain nombre de participants impliqués dans le programme du $2^{\mathrm{e}}$ cycle ont jugé le terme «peu 
accessible » aux élèves de cet âge et en ont retardé la présentation. Par ailleurs, l'usage habituel - et non véritablement remis en cause - du terme de groupe verbal (GV), aux $1^{\mathrm{er}}$ et $2^{e}$ cycles, pendant de longues années, pour désigner à la fois la classe grammaticale et la fonction du GV suffit sans doute à expliquer ces faits. Le plan d'études romand est actuellement disponible dans une double version, papier et électronique. Lors de sa publication, il a été clairement affirmé qu'il s'agissait d'un texte révisable et évolutif. Peutêtre l'introduction du terme de prédicat constituera-t-elle une des premières manifestations visibles de son évolutivité...

Dans la grammaire de référence, destinée aux enseignant.e.s ainsi que dans les mémentos Texte et Langue (Aubert \& Messeiller, 2015 ; Balma \& Roduit, 2010) prévus pour les élèves des $2^{\mathrm{e}}$ et $3^{\mathrm{e}}$ cycles, le terme de prédicat est présent. Dans Lire, écrire, comprendre la grammaire et la langue (Balma, Tardin \& de Pietro, 2013), ce terme est d'abord explicitement défini comme l'un des deux constituants obligatoires de la phrase, avec le sujet. Il apparait également dans le cadre d'une étude systématique des fonctions grammaticales: la fonction prédicat est celle du groupe verbal et ses caractéristiques syntaxiques sont énoncées :

- le prédicat est un élément essentiel de la phrase ;

- on ne peut ni le supprimer ni le déplacer avant le sujet ;

- le verbe du prédicat s'accorde en personne et en nombre avec le sujet, parfois en genre pour le participe passé (ibid.).

Le plan retenu pour rendre compte de la fonction prédicat est exactement identique au plan suivi pour toutes les autres fonctions. Ce parti pris de présentation participe à la valorisation de la fonction prédicat en tant qu'élément d'un "système " cohérent: la classe grammaticale GV a une fonction, celle de prédicat.

Dans les moyens d'enseignement romands, pour les $2^{\mathrm{e}}$ et $3^{\mathrm{e}}$ cycles, il n'est pas question de prédicat. Il faut préciser que les quatre collections concernées - Mon Manuel de français, L'île aux mots pour le $2^{\mathrm{e}}$ cycle ; L'atelier du langage, Français, livre unique pour le $3^{\mathrm{e}}$ datent du début des années 1990 pour la plus ancienne (L'île aux mots) et de 2002 pour la plus récente (Mon Manuel de français). Ces collections sont « importées » de France ${ }^{3}$ et, même si elles ont été "romandisées", elles portent la trace des conceptions qui les fondent... S'agissant du prédicat, le terme n'est pas présent parce que la tradition française privilégiait le plus souvent à l'époque une analyse de la phrase qui fait du verbe le pivot autour duquel s'organisent les actants que sont le sujet, les compléments attendus ou impliqués par ce verbe - qui, selon certaines traditions s'appellent des compléments d'objet direct (COD), des compléments d'objet indirect (COI), des compléments d'objet second (COS) ou encore des compléments de verbe (CV) direct ou indirect. Les compléments circonstanciels ou encore compléments de phrase (CP) quand bien même les deux désignations ne recouvrent pas vraiment les mêmes réalités syntaxiques sont présentés comme des compléments non essentiels, incidents à la phrase de base. Cette analyse s'inscrit dans la filiation des propositions de L. Tesnière et se retrouve de manière explicite dans les perspectives développées dans la collection Mon Manuel de français (2002), initiées par B. Couté et reprises par F. Rondelli dans la suite de la collection.

$\mathrm{Au}$ moment de l'adaptation pour la Suisse romande des collections françaises choisies pour le $2^{\mathrm{e}}$ cycle, très peu de temps avant la publication du PER (2010), il n'était pas question de prédicat, ni dans les pratiques romandes - qui parlaient de groupe nominal sujet et de groupe verbal - ni dans les pratiques françaises, intéressées par la construction du verbe et non préoccupées par le groupe verbal. Pourtant, des manques et incohérences 
par rapport à un système que l'on souhaitait cohérent ont été pointés (Panchout-Dubois \& Tardin, 2010). Concernant le système de désignation des fonctions, l'absence de fonction pour le GV dans le système terminologique français et suisse romand a été signalée comme problématique : le non-usage de la fonction de "prédicat » nuit à l'intelligibilité de la distinction fondamentale en grammaire scolaire entre «classe» et «fonction ». $\mathrm{M}$. Panchout-Dubois et $\mathrm{C}$. Tardin ont aussi signalé le caractère très dommageable de la disparition de la classe grammaticale groupe prépositionnel (GPrép) au profit de groupe nominal avec préposition (GN avec Prép). En outre, ils ont critiqué l'absence d'une appellation claire pour désigner la fonction des déterminants, laquelle ajoute encore du flou à une construction déjà fragile.

\section{Enquête sur les pratiques d'enseignement : propos rapportés d'étudiant.e.s stagiaires et de responsables de formation}

23 À la HEP, en formation initiale, les étudiant.e.s abordent les contenus relatifs au fonctionnement de la langue dès leur premier semestre de formation ; cinq cours d'1h30, intégrés à un module intitulé Lire et écrire; savoirs fondamentaux et gestes professionnels, visent à les introduire aux savoirs linguistiques. C'est au cours de la deuxième année de formation (qui en comporte 3), dans le cadre du module Fonctionnement de la langue orale et écrite que des temps spécifiques sont consacrés à la grammaire, au lexique et à l'orthographe, tantôt sous forme de cours magistraux, tantôt sous forme de travaux dirigés. Comment le terme de prédicat apparait-il dans ce cadre?

24 Au moment de l'abord de la phrase en tant qu'unité syntaxique, le terme de prédicat apparait, indissociablement lié à celui de sujet. Il est défini comme l'un des deux constituants fondamentaux de la phrase et, en contraste avec ces deux constituants obligatoires, sont identifiés les éventuels constituants facultatifs. Au sein d'énoncés constitués en corpus, les étudiant.e.s prennent conscience des substitutions réalisables sur l'axe paradigmatique, définissant la notion de classe grammaticale et des relations existant entre les groupes de mots et les mots, définissant la notion de fonction syntaxique. La fonction prédicat fait l'objet d'une étude systématique. Ainsi, les différents types de prédicat sont analysés :

- prédicat réduit au seul verbe,

- prédicat comportant un verbe et un complément de verbe, direct ou indirect,

- prédicat comportant un verbe et deux compléments de verbe, direct et indirect, ou indirects tous deux,

- prédicat comportant un verbe suivi d'un attribut du sujet ou d'un complément de verbe suivi d'un attribut du complément de verbe.

C'est grâce au concours des étudiant.e.s inscrits au module Fonctionnement de la langue orale et écrite au semestre du printemps 2017 que nous avons pu mener une enquête autour de l'usage du terme de prédicat dans les pratiques d'enseignement vaudoises. Les quelque 250 étudiant.e.s engagé.e.s dans le module et effectuant un stage en école maternelle et primaire devaient demander aux enseignant.e.s qui les accueillaient quel usage était fait du terme de prédicat dans la réalité de leur classe, et comment ils justifiaient les choix effectués. Ils devaient également leur demander d'expliciter quelle relation eux-mêmes entretenaient avec le terme. Les résultats de cette enquête donnent d'intéressantes indications sur le non-usage du terme. Sur les 188 réponses effectives, en mettant de côté les classes de l'école enfantine de $1^{\text {re }}$ et de $2^{\mathrm{e}}$ primaire (près de 60 classes), 
une belle unité apparait concernant le non-usage du terme avec les élèves. La plupart du temps, les enseignant.e.s du $2^{\mathrm{e}}$ cycle soulignent que les prescriptions du plan d'études ne mentionnent pas le prédicat et ajoutent souvent que le terme ne leur parait pas très transparent. Certains d'entre eux - près des $2 / 3$ (enseignants de l'école enfantine inclus) - disent explicitement ne pas avoir connaissance du mot. Ceux qui en ont connaissance pensent qu'il est prudent, dans un souci de recevabilité, d'en différer la présentation.

Et dans les autres cantons? Nous avons interrogé quelques collègues, responsables de la formation des enseignants primaire et secondaire, dans les divers cantons suisses romands. À Genève, bien que le discours de la formation promeuve le terme, les pratiques enseignantes et le flottement de l'usage du terme dans les divers documents officiels confortent l'usage du terme de GV. Dans le canton de Fribourg, malgré l'usage du terme dans le mémento grammatical pour le $3^{\mathrm{e}}$, le terme de GV est de toute évidence plus usité que celui de prédicat dans les supports de classe. Les formateurs expliquent cet état des choses par l'absence du terme dans le PER aux $1^{\text {er }}$ et $2^{\mathrm{e}}$ cycles. Ils sont aussi d'avis que, pour l'étudiant.e débutant, la notion de GV est bien plus ancrée aux savoirs de base que celle de prédicat. Les formateurs disent en parler en cours, notamment pour expliquer la confusion entre classe et fonction à propos du GV et voient donc l'intérêt de la notion. Pour certains, la problématique du prédicat n'est que la pointe de l'iceberg de l'emploi d'un métalangage inapproprié... En Valais, pour le cycle 3, la notion de prédicat n'est que très peu utilisée. Les formateurs expliquent le non-usage par le fait que le terme n'est pas utilisé dans les moyens d'enseignement officiels; c'est le terme de GV qui prévaut au moment de l'analyse des fonctions de la phrase. La situation est plus optimiste dans le canton de Neuchâtel où une fiche guide a été élaborée et diffusée pour clarifier la terminologie de la grammaire de la phrase pour l'ensemble de la scolarité obligatoire ${ }^{4}$. Cette mesure et l'introduction de la notion dans le mémento pour le $3^{\mathrm{e}}$ cycle semblent accroitre la diffusion de la notion. D'après une formatrice à la HEP Béjune, le prédicat est désormais enseigné aux élèves de $7^{\mathrm{e}}$ année ; c'est ainsi que les élèves nomment la fonction $\mathrm{du} \mathrm{GV}$ en arrivant dans les classes.

\section{Pourquoi adopter le terme de prédicat dans le cadre d'une grammaire scolaire?}

Les raisons de l'adoption du terme dans le cadre d'une grammaire scolaire sont en lien étroit avec les objectifs qui organisent l'enseignement grammatical. Si l'un de ces objectifs est bien de construire la langue en tant que système, la distinction entre classe grammaticale et fonction syntaxique est fondatrice. Dans cette perspective, comment pourrait-on se priver d'un terme spécifique pour désigner la fonction du groupe verbal?

Dans la lignée de S.-G. Chartrand (2009) qui développe explicitement les raisons de promouvoir le prédicat de la phrase, il faut militer pour un usage du terme en grammaire scolaire. Il n'est en effet pas possible d'imaginer qu'il n'y ait pas d'étiquette pour nommer la fonction du groupe verbal.

Il est intéressant de constater que le «manque » de mot pour désigner la fonction du groupe verbal, apparait de manière saisissante dans des schémas tels que celui reproduit ci-dessous, extrait d'Ouvrir la grammaire (Genevay, 1994), un ouvrage pensé pour les élèves du secondaire 1 . 
Figure 1. Schéma extrait d'Ouvrir la grammaire d'É. Genevay (1994, p. 50)

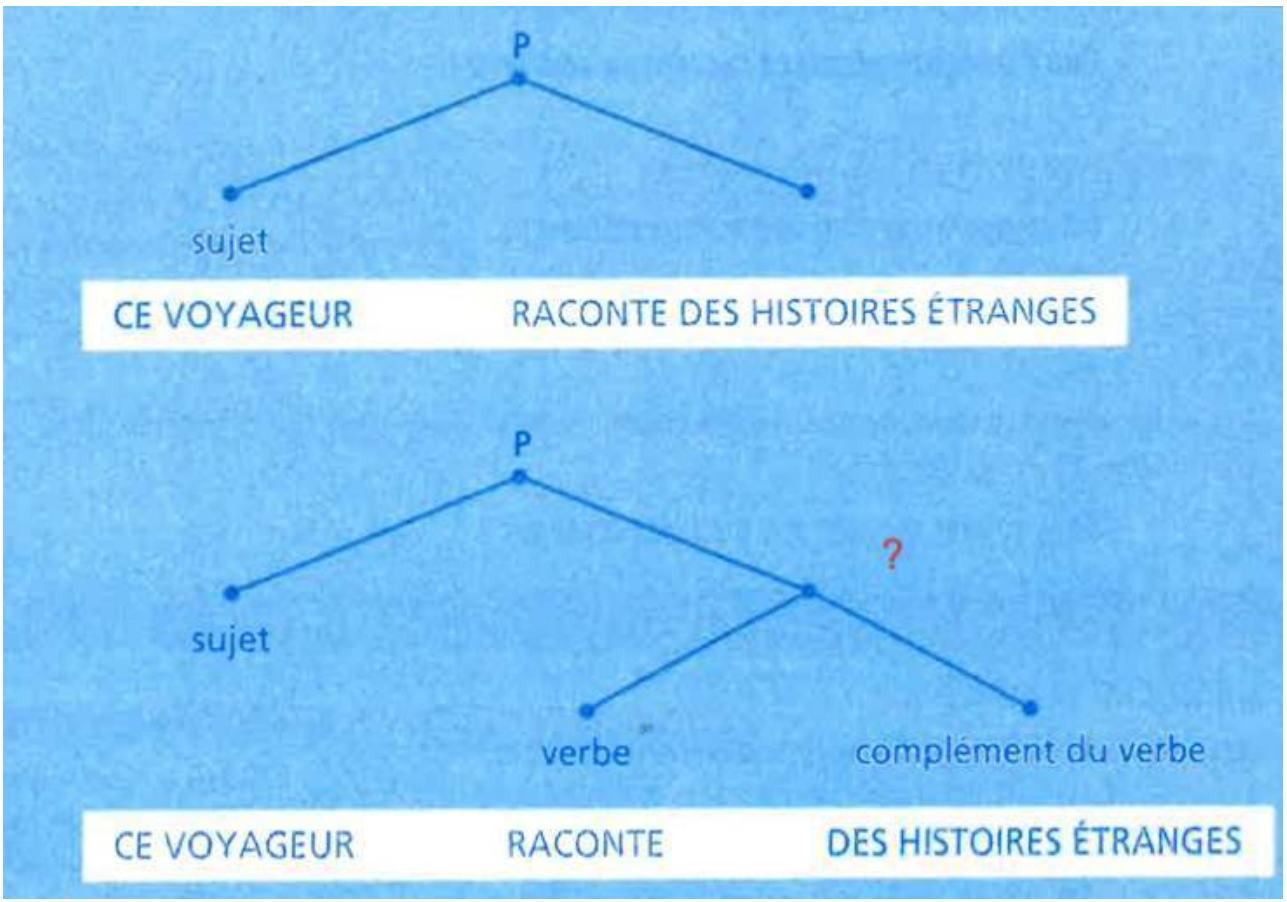

Le point d'interrogation est évidemment de notre cru...

Ce qu'il faut peut-être remarquer, c'est le caractère un peu paradoxal de " combler » une absence de terme désignant une relation syntaxique dans un système qui se veut formel par un terme d'origine logico-sémantique. Par ailleurs, C. Vargas (1995) discute de la relative inadéquation de l'analyse en sujet + prédicat lorsqu'il s'agit de faire percevoir l'accord qui existe entre le sujet et le verbe, noyau du prédicat ; on ne peut parler d'accord sujet-prédicat, mais d'accord sujet-verbe. De la même manière, lorsqu'une phrase présente une incompatibilité sémantique entre le verbe de la phrase et son sujet, le terme de prédicat fait obstacle puisque l'on ne peut parler d'incompatibilité entre le sujet et le prédicat, mais d'une incompatibilité entre le sujet et le verbe de ce prédicat.

2 Cette analyse autour du terme de prédicat met en évidence l'urgence d'une réflexion sur les choix terminologiques tant il est vrai qu'ils sont les signes visibles d'une conception $\mathrm{du}$ fonctionnement de la langue et les vecteurs de l'intelligibilité des systèmes d'explication de la langue (Panchout-Dubois \& Tardin, 2010). À ce propos, on peut rêver d'une reprise des travaux d'harmonisation de la terminologie grammaticale à l'échelle francophone.

\section{Et pour demain?}

Une mesure prioritaire semble s'imposer: une révision du PER qui proposerait explicitement le prédicat comme l'un des constituants obligatoires de la phrase dès le $1^{\mathrm{er}}$ et $2^{\mathrm{e}}$ cycle et qui mettrait au programme une étude systématique des fonctions, dont celle de prédicat. Il reste ensuite à espérer une rapide décision politique visant la production de moyens d'enseignement romands réellement en prise avec les prescriptions d'un plan d'études actualisé. 

systématique dans les institutions de formation suisses romandes. Cet état de fait conforte le constat fait ailleurs (Gagnon \& Laurens, 2016) selon lequel les pratiques professionnelles de terrain l'emportent sur les contenus et démarches proposés en formation. Ce qui appelle la mise en place d'actions concertées dans le cadre de la formation continue : les communautés d'apprentissages communautaires, les recherches action ou collaboratives constituent sans doute des espaces indispensables à la promotion du changement.

Il reste un problème de taille à gérer au sein même de la formation initiale : celui de la didactisation, à l'adresse des étudiant.e.s, de tous les éléments d'élucidation des niveaux d'analyse mis en œuvre dans le cadre de la réflexion grammaticale: sémantique, énonciatif, morphosyntaxique (Vargas, 1995). C'est à ce prix que les vœux du Père Girard, en épigraphe à ce texte, seront exaucés.

\section{BIBLIOGRAPHIE}

AUBERT, Fl. \& MESSEILLER, Ch. (2015). Texte et langue ; Aide-mémoire, savoirs grammaticaux et ressources théoriques pour les élèves du cycle 2. Neuchâtel : CIIP (Conférence intercantonale de l'instruction publique de la Suisse romande et du Tessin).

BALMA, P.-A. \& RODUIT Ph. (2010). Texte et langue. Aide-mémoire, savoirs grammaticaux et ressources théoriques pour les élèves du cycle 3. Neuchâtel : CIIP.

BALMA, P.-A., TARDIN, Ch., DE PIETRO, J.-F. (2013). Lire, écrire, comprendre la grammaire et la langue. Neuchâtel : CIIP. En ligne : http://www.spsressources.ch/wordpress/wp-content/ uploads/2013/11/grammairereference.pdf.

BESSON, M.-J. et al. (1979). Maîtrise du français. Lausanne : Office romand des Éditions et du matériel scolaires.

BRONCKART, J.-P. \& SZNICER, G. (1990). « Description grammaticale et principes d'une didactique de la grammaire ». Le français aujourd'hui 89, p. 5-16. En ligne : https://archive-ouverte.unige.ch/ unige:37341.

BULEA BRONCKART, E. (2015). Didactique de la grammaire. Genève : Université de Genève.

CHARTRAND, S. \& DE PIETRO, J.-F. (2012). « Pour une harmonisation des terminologies grammaticales scolaires de la francophonie : quels critères pour quelles finalités ? » Enjeux 84, p. 5-31.

CHARTRAND, S.-G. (2009). « Cachez ce prédicat que je ne saurais voir ». Correspondance 15, 2. En ligne : http://correspo.ccdmd.qc.ca/index.php/document/des-racines-et-des-ailes/cachez-cepredicat-que-je-ne-saurais-voir/.

CHERVEL, A. (1977). Et il fallut apprendre à écrire à tous les petits français. Histoire de la grammaire scolaire. Paris : Payot. 
CIIP. (2010). Plan d'études romand. Neuchâtel : Conférence intercantonale de l'instruction publique de la Suisse romande et du Tessin.

GAGNON, R. \& LAURENS, V. (2016). « Circulation de savoirs entre institution de formation et terrains scolaires : analyse de dispositifs de formation à l'enseignement de la production écrite en Suisse romande ». Phronesis 5, 3-4, p. 69-86.

GENEVAY, É. (1994). Ouvrir la grammaire. Lausanne : LEP.

GIRARD, Gr. (1876). Cours éducatif de langue maternelle pour les écoles et les familles. Paris : Delagrave.

GRÉVISSE, M. (1980) [1936]. Le Bon usage. Paris : Duculot.

LE GOFFIC, P. (1993). Grammaire de la phrase française. Paris : Hachette.

PANCHOUT-Dubois, M. \& TARDIN, C. (2010). « Quelle compatibilité entre les traditions

grammaticales des différents pays francophones? L'exemple de l'adaptation de moyens

d'enseignement français en Suisse romande ». AIRDF, la lettre de l'association 45-46, p. 53-58.

PELLAT, J.-Ch. (éd.) (2011) [2009]. Quelle grammaire enseigner ? Paris : Hatier.

SCHNEUWLY, B. \& DOLZ, J. (2009). Des objets enseignés en classe de français. Le travail de l'enseignant sur la rédaction de textes argumentatifs et sur la subordonnée relative. Rennes : Presses universitaires de Rennes.

VARGAS, C. (1995). Grammaire pour enseigner. T. 2. La phrase verbale, fonctions et catégories grammaticales. Paris : A. Colin.

\section{NOTES}

1. A. Chervel (1977, p. 21) affirme d'ailleurs que cette nouvelle syntaxe dite « des fonctions » fait de la grammaire scolaire « le champ clos des tours de passepasse ». P. Le Goffic souligne d'ailleurs qu'une phrase telle que «Il y a un carreau de cassé » n'est pas analysable avec ce système de fonctions.

2. En Suisse, le premier cycle comprend la $1^{\text {re }}$ à la $4^{\mathrm{e}}$ année primaire (élèves de 4 à 8 ans), le $2^{\mathrm{e}}$ cycle comprend la $5^{\mathrm{e}}$ à la $8^{\mathrm{e}}$ année primaire (élèves de 9 à 12 ans) et le $3^{\mathrm{e}}$ cycle intègre les trois premières années du secondaire obligatoire (élèves de 12 à 15 ans).

3. Il y aurait beaucoup à dire aussi sur les raisons, politiques et économiques, qui ont motivé les choix de ces moyens d'enseignement.

4. La fiche est disponible à l'adresse suivante : https://portail.rpn.ch/beo/Biblio/ResourcesList/ fiche_guide_grammaire_phrase.pdf.

\section{RÉSUMÉS}

L'article propose un état des lieux de l'enseignement grammatical en Suisse romande avec une focale sur l'usage du terme de prédicat. Nous regardons d'abord les reformulations successives du terme au cours de l'histoire. Puis, grâce à une analyse des prescriptions institutionnelles, aux résultats d'une enquête menée par nos étudiants auprès des responsables de stage, à des 
témoignages de formateurs des divers cantons romands, nous mettons en évidence un écart entre les savoirs de la formation et les pratiques déclarées. Cet état des lieux nous conduit à formuler un argumentaire en faveur de l'usage du terme de prédicat dans la grammaire scolaire et à envisager quelques pistes pour l'effectivité de son usage dans les classes.

This contribution gives an overview of grammar teaching in French Switzerland and highlights the use of a specific notion: the predicate. After a brief historical perspective and the presentation of the official documents (curriculum and others official teaching resources), we discuss the results of an inquiry on the use of the notion of predicate in French Swiss teaching practices. Based on all these elements, we develop arguments that state our position on the necessary use of the notion in order to give a systematic view of the functioning of French language. We also suggest some avenues to explore in teacher training programmes.

\section{INDEX}

Mots-clés : prédicat, groupe verbal, classe grammaticale, fonction syntaxique, pratiques d'enseignement, formation des enseignants

Keywords : predicate, verbal group, grammatical category, syntactic function, teaching practices, teacher education

\section{AUTEURS}

\section{MARTINE PANCHOUT-DUBOIS}

Haute École Pédagogique, $\mathrm{CH}-1014$, Suisse

\section{ROXANE GAGNON}

Haute École Pédagogique, $\mathrm{CH}-1014$, Suisse

ECATERINA BULEA BRONCKART

Université de Genève, FPSE, CH-1014, Suisse 